\title{
Cardiovascular Reactivity after Bilateral Nephrectomy in Rats
}

\author{
Hirofumi Sokabe, M.D., Fumio Shibayama, B.P., \\ Susumu Mizogami, B.P., and Fuminori Sakai, M.D.
}

Cardiovascular reactivity was determined 16 to 28 hours after bilateral nephrectomy by the dose-blood pressure-response to pressor and depressor agents. There was no increase in reactivity to pressor agents after bilateral nephrectomy in rats anesthetized with pentobarbital or treated by pentolinium, but pithed animals without anesthesia showed an increased response. Elimination of the neural innervation in the pithed rat by atropine, bretylium, and pentolinium also showed an increased sensitivity to angiotensin. Evidence is presented that the supersensitivity observed and the large increase in angiotensinogen content of the plasma is responsible for the marked augmentation of response to renin after nephrectomy.

$\mathrm{B}$ ILATERAL nephrectomy augments pressor response to renin, as first shown by Tigerstedt and Bergman in 1898.1) The response to other pressor agents is less well established. Hoobler et al. ${ }^{2)}$ reported that the response to norepinephrine and angiotensin remained unchanged after bilateral nephrectomy in rats treated with pentolinium, a ganglion-blocking agent. They attributed the augmentation in the pressor response to renin to the appearance in the plasma following nephrectomy of an incubation product of renin other than the ordinary angiotensin. They subsequently excluded changes in angiotensinase, converting enzyme, renin binding capacity, or the presence of inhibitors or accelerators of the renin-angiotensinogen reaction ${ }^{3)}$ as responsible for the increased pressor response. The purpose of the present study is to re-examine this problem. For the determination of cardiovascular reactivity, the dose-blood pressure-response curves were obtained to avoid the vertical bias, as shown by Trendelenburg." The term " cardiovascular reactivity" is used as defined by Page and Bumpus. ${ }^{5}$ " The dose-response curves were determined not only to pressor agents but to depressor agents. An appreciable effect of bilateral nephrectomy on the responses to the latter has not hitherto been reported.

From the Department of Pharmacology, Faculty of Medicine, University of Tokyo, Tokyo. Part of this work was supported by a Grant from the International Cardiology Foundation. 


\section{Methods}

Rats of the Donryu strain, of either sex, weighing 200 to $300 \mathrm{Gm}$. were used. Bilateral nephrectomy was performed through a flank incision under ether anesthesia 16 to 28 hours before the test. In preliminary experiments using 17 each of bilaterally nephrectomized and sham operated rats, responses to a single dose of semipurified rat renin $(0.1 \mathrm{ml}$. of $1 / 6$ dilution, i.v.) were determined at several time intervals $(1,2,4,8,12,16,24$ and 28 hours after operation). The sensitivity was increased 12 to 28 hours after nephrectomy but not before 8 hours. These results differed from those reported by Gross. ${ }^{6)}$ Sham operation did not affect the sensitivity significantly. Therefore, intact animals of comparable weight and sex were used as controls, unless otherwise noted.

For the determination of dose-blood pressure-response curves, ${ }^{7}$ the rats were anesthetized by pentobarbital sodium ( $40 \mathrm{mg}$./Kg., i.p., for the nephrectomized rats and $50 \mathrm{mg} . / \mathrm{Kg}$. for the controls), heparinized (100 units $/ \mathrm{Kg}$. of heparin sodium, Taiyo Fishery) and tracheotomized. The right femoral artery was cannulated with a fine polyethylene tubing, through which the blood pressure was recorded by a recording system (Statham transducer P23AA, Sanborn recorder 151-100A, 150-1100AS and 150-200B/400). The left femoral vein was cannulated for intravenous injection. The internal volume of the cannula was about $0.025 \mathrm{ml}$. All drugs were dissolved in $0.9 \%$ saline to a volume of $0.1 \mathrm{ml}$. Immediately after the injection the venous cannula was flushed with $0.05 \mathrm{ml}$. of salinc. The following drugs were used: synthetic asparagine ${ }^{1}$-valine ${ }^{5}$-angiotensin II (Hypertensin, Ciba), dl-norepinephrine bitartrate monohydrate (the doses refer to one half of the free base), acetylcholine chloride, adenosine triphosphoric acid disodium salt (Kowa), and histamine dihydrochloride (the doses of the latter substances refer to the salt). The blood pressure was maintained at 90 to $120 \mathrm{~mm} \cdot \mathrm{Hg}$ by varying the dose of anesthetic, since the pressor response to an amount of drug is inversely related to the original blood pressure level with a correlation coefficient of $0.94 .^{87}$ For ganglion blockade, pentolinium tartrate (Ansolysen, Wyeth) was utilized ( $5 \mathrm{mg} . / \mathrm{Kg}$., i.v.), the blood pressure being maintained at 60 to $80 \mathrm{~mm} . \mathrm{Hg}$. The rats were pithed by inserting a steel rod $(2.4 \mathrm{~mm}$. in diameter) with a sharpened tip through the left orbital fossa to the end of spinal cannal. Previous to pithing, the animal was anesthetized with ether, and cannulations and heparinization were carried out. The tracheal cannula was connected to a positive-pressure respirator (C. F. Palmor, Ltd.). Following pithing at least $20 \mathrm{~min}$. was allowed to elapse to remove the ether. In the pithed animals, further elimination of neural innervation was achieved by bretylium tosylate of Welcome Research Laboratories, Kent (30 mg./Kg., i.v.), atropine sulfate ( $2 \mathrm{mg} . / \mathrm{Kg}$., i.v.) and pentolinium tartrate (5 mg./Kg., i.v.).

For the determination of angiotensionogen, the plasmas were obtained without hemolysis by collecting the blood with heparin through polyethylene cannula inserted into the carotid artery. It was cooled immediately and centrifuged at 3,000 r.p.m. for $15 \mathrm{~min}$. One ml. of the plasma was incubated at $37^{\circ} \mathrm{C}$ for $10 \mathrm{~min}$. with $1 \mathrm{ml}$. of semipurified rat renin and with $1 \mathrm{ml}$. of phosphate buffer $(\mathrm{pH} 6.5$ ) containing $0.002 \%$ thimerosal. The reaction was stopped by boiling the incubation mixture for 5 min., and centrifuged. The supernatant was used for assay. 
The semipurified rat renin was prepared by extracting the kidneys frozen at $-20^{\circ} \mathrm{C}$ with cold glass-distilled water $\left(0^{\circ} \mathrm{G}, 1 \mathrm{ml} / / \mathrm{Gm}\right.$. of tissue). After grinding, it was centrifuged at $0^{\circ} \mathrm{C}, 12,000$ r.p.m. for $15 \mathrm{~min}$. The supernatant was acidified to $\mathrm{pH} 3.0$, incubated at $0^{\circ} \mathrm{C}$ for $30 \mathrm{~min}$., and the $\mathrm{pH}$ was brought back to 7.4 and centrifuged. It was then dialysed against cold water overnight. Sodium chloride was added to make a $0.9 \%$ solution. This preparation was free from heat-stable pressor substances, and its angiotensinase content was considerably decreased. Angiotensinase activity in the plasmas was determined by incubating at $37^{\circ} \mathrm{C}$ for $10 \mathrm{~min}$. with standard angiotensin II (Hypertensin, Ciba) solution $(0.4 \mu \mathrm{g} . / \mathrm{ml}$.) of $\mathrm{pH}$ 6.5. It was compared with the pressor activity of the mixture in which the plasma had been boiled for $5 \mathrm{~min}$. Angiotensin in the incubation mixture was determined by its pressor action in female rats (200 to $225 \mathrm{Gm}$.) treated as same as the above pentolinium-treated preparation. The effect of $0.3 \mathrm{ml}$. of the mixture was compared with a standard angiotensin solution containing 0.005 to $0.32 \mu \mathrm{g} . / 0.3$ $\mathrm{ml}$. Details of the method are described elsewhere. ${ }^{9)}$

\section{REsULts}

Effect of bilateral nephrectomy on cardiovascular reactivity to pressor agents Doseblood pressure-response curves of angiotensin (Ang) and norepinephrine (NE) obtained in the bilaterally nephrectomized rats were compared with those of the normal controls. Under pentobarbital anesthesia, cardiovascular reactivity of nephrectomized animals to Ang or NE was not increased over the normal (Fig. 1). In fact, the normal rats appeared more sensitive than the nephrectomized. In these experiments the amount of pentobarbital was controlled so as to have the same blood pressure level in both the nephrectomized and the control rats. Less anesthetic was generally used in the former as mentioned under methods. If the same amount had been used, the blood pressure level of the bilaterally nephrectomized animals would be lower, because they are more sensitive to pentobarbital. Consequently, the responses
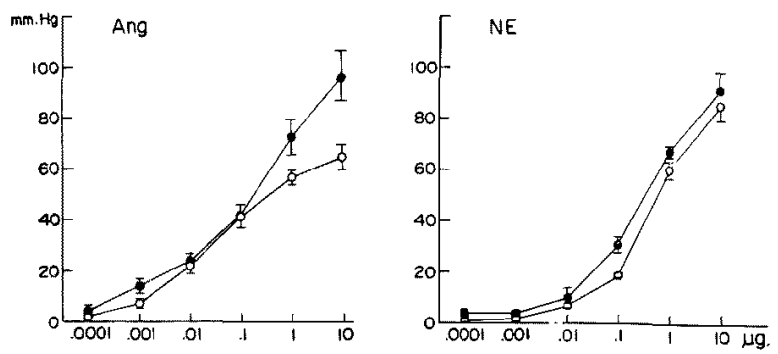

Fig. 1. Effect of bilateral nephrectomy on cardiovascular reactivity to pressor agents in rats. Mean response of 6 normal (- -) and of 6 nephrectomized animals (-O-). The standard errors of the means are indicated as vertical bars. Ordinate: blood pressure responses in $\mathrm{mm}$. $\mathrm{Hg}$, abscissa: doses of the agents per rats given intravenously. 
to the pressor agents would become larger by the reason described above. ${ }^{8}$ This was presumably the case when increased responses were noted in the past.

Cardiovascular reactivity was also determined using a single dose of Ang (0.1 $\mu$ g., i.v.) and NE $(0.3 \mu$ g., i.v. $)$, which elicited a response about $50 \%$ of the maxima (Fig. 1). This reconfirmation was necessary because we found that the previous injection of a drug had influenced the following response and that injection of drugs in the ascending or descending order of doses showed a different result when the dose-blood pressure-response curve was obtained. However, it never invalidates to obtain the full dose-response curve in order to avoid the vertical bias." In each of 5 experiments, the responses of $54 \pm$ $1.7 \mathrm{~mm}$. $\mathrm{Hg}$ to Ang and of $46 \pm 3.6$ to $\mathrm{NE}$ in the normal, and of $49 \pm 2.9$ to Ang and of $42 \pm 1.4$ to NE in the nephrectomized were obtained. Figures are mean \pm standard error. Statistical analysis revealed that the differences were not significant.

Dose-blood pressure-response curves of Ang and $\mathrm{NE}$ obtained in the rats treated with pentolinium are shown in Fig. 2. Bilateral nephrectomy did not change the cardiovascular reactivity to the agents. Thesc results are similar to those reported by Hoobler et al. ${ }^{2)}$
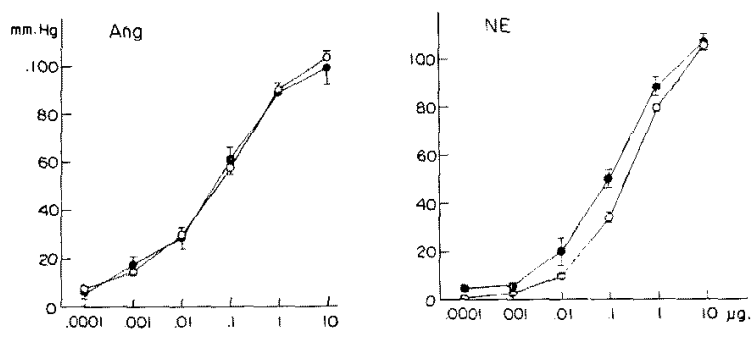

Fig. 2. Effect of bilateral nephrectomy on cardiovascular reactivity to pressor agents in rats treated with $5 \mathrm{mg} / \mathrm{Kg}$. of pentolinium tartrate intravenously. Mean response of 5 normal (--) and of 7 nephrectomized rats $(-\mathrm{O} \rightarrow$ ). Presentation as in Fig. 1.

Although administration of the ganglion blocking agent may be expected to remove the cardiovascular reflexes to a certain degree, the effect is not complete and the preparation requires the use of general anesthetics. Therefore, observations on the pithed animal without anesthesia were also carried out. The dose-blood pressure-response curves are shown in Fig. 3. After pithing, the nephrectomized rats were slightly more sensitive than the normal to the pressor agents. In contrast response to renin was markedly augmented by bilateral nephrectomy in pithed animals, as well as under usual condition. In each of 4 experiments, injection of semipurified rat renin $(0.3 \mathrm{ml}$, of $1 / 5$ 

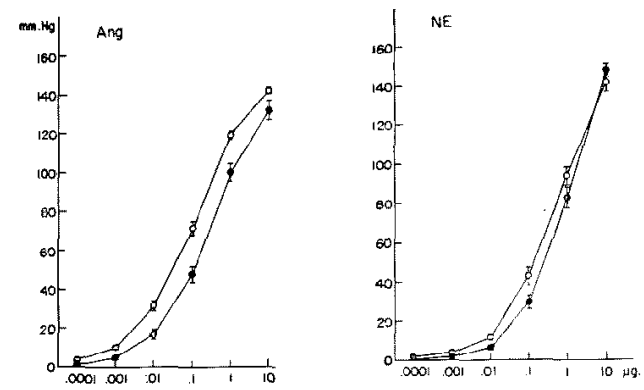

Fig. 3. Effect of bilateral nephrectomy on cardiovascular reactivity to pressor agents in pithed rats. Sixteen normal (- - ) and 15 nephrectomized animals (-O-).

dilution, i.v.) elicited a response of $15.0 \pm 3.5 \mathrm{~mm} . \mathrm{Hg}$ in the normal and of $71.0 \pm 5.7$ in the nephrectomized preparation.

Responses to Ang also increased in each of 5 preparations of pithed rats in which neural innervation was minimized by administration of bretylium, atropine and pentolinium. Bilateral nephrectomy thus augments cardiovascular reactivity to Ang or NE slightly, but it is inadequate to explain the marked augmentation of response to renin after nephrectomy.

Effect of bilateral nephrectomy on cardiovascular reactivity to depressor agents Dose-blood pressure-response curves of acetylcholine ( $\mathrm{ACh})$, adenosine triphosphate (ATP), and histamine (Hist) were obtained in the rat anesthetized with pentobarbital only and treated with pentolinium (Fig. 4 and 5). Bilateral nephrectomy did not affect obviously the response to ACh or ATP. Response to Hist were greatly reduced by nephrectomy. However, in the experiment using the single dose of ACh (0.1 $\mu$ g., i.v.), ATP (30 $\mu$ g., i.v.), and Hist $(30 \mu \mathrm{g} .$, i.v.), the differences in the responses became more apparent. The reason why we did this reconfirmation is already given in the previous section. Each of 4 experiments was carricd out with 3 injections at each dose level. The doses were chosen so as to elicit a response about $50 \%$ of the maxima from the dose-response curves (Fig. 4). The exact figures were $53 \pm 2.7 \mathrm{~mm} . \mathrm{Hg}$ and $34 \pm 2.6 \mathrm{~mm}$. $\mathrm{Hg}$ to $\mathrm{ACh}, 55 \pm 6.7 \mathrm{~mm}$. $\mathrm{Hg}$ and $40 \pm 3.4$ $\mathrm{mm}$. $\mathrm{Hg}$ to ATP, and $56 \pm 4.0 \mathrm{~mm}$. $\mathrm{Hg}$ and $30 \pm 5.6 \mathrm{~mm}$. Hg to Hist. The
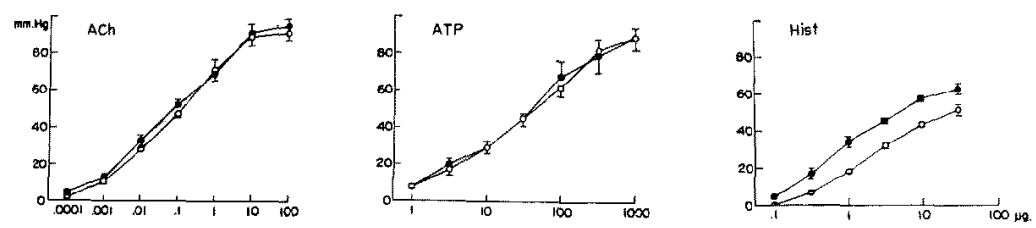

Fig. 4. Effect of bilateral nephrectomy on cardiovascular reactivity to depressor agents. Each 7 of normal (- -) and nephrectomized rats (-O-). 

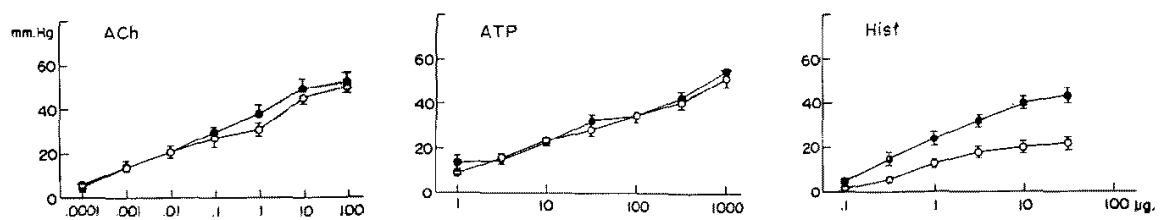

Fig. 5. Effect of bilateral nephrectomy on cardiovascular reactivity to depressor agents in rats treated with pentolinium. Each 6 of normal (- -) and nephrectomized rats (-O-) were observed.

formers refer to the normal controls and the latters to the nephrectomized rats, respectively. The differences are statistically significant $(\mathrm{P}<0.05)$.

Effect of bilateral nephrectomy on angiotensinogen content in the plasma Although many have reported an increase in angiotensinogen after bilateral nephrectomy, ${ }^{101-12)}$ it was pertinent to determine if a further increase in concentration of angiotensinogen would lead to an increased production of angiotensin when it was already in excess, which Hoobler et al. ${ }^{21}$, 3) have claimed was not likely. The experiments were carried out by incubating semipurified rat renin in various concentration ( $1 / 300$ to $1 / 1)$ with plasma of 7 normal control rats, 5 sham operated 0 to 2 hours before, 5 sham operated 16 to 28 hours before, 5 bilaterally nephrectomized 0 to 2 hours before, and 5 nephrectomized 16 to 28 hours before (Fig. 6). After incubation for $10 \mathrm{~min}$., formation of angiotensin was determined and expressed in $\mu \mathrm{g}$. per $\mathrm{ml}$. of the plasmas added. The plasma from the rats bilaterally nephrectomized 16 to 28 hours before yielded $2.4 \mu \mathrm{g}$. of angiotensin per ml. in the maximum. This was 4 times more than the other plasmas. The control values were 0.54 to $0.62 \mu \mathrm{g} . / \mathrm{ml}$., in-

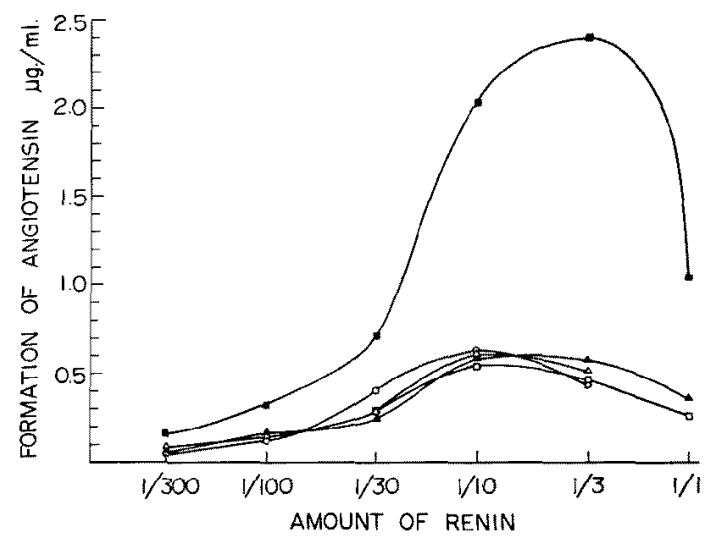

Fig. 6. Effect of bilateral nephrectomy on angiotensinogen contents in the plasma. Various amount of renin was incubated with the plasmas of normal control ( $-\mathrm{O}-$ ), sham operated rats 0 to 2 hours before $(-\Delta-)$, and 16 to 28 hours before (-A-), bilaterally nephrectomized rats 0 to 2 hours before ( $\square-$ ), and 16 to 28 hours before $(-\mathbf{m}-)$. 
dicating that sham operation or nephrectomy 0 to 2 hours before did not change the angiotensinogen content of the plasma. Decreased angiotensin formation at the higher concentration of renin was due to the presence of angiotensinase in the renin preparation and in the plasma. Angiotensinase activity of 5 plasmas were in the same range. Reaction constants calculated from the curve and converted at the same enzyme concentration were also in the same range, as reported by Hoobler et al. ${ }^{3}$ )

The important findings in these experiments were differences of angiotensin formation at renin concentrations less than $1 / 10$. At these concentrations, angiotensinogen was in excess in either case. But the plasma from the bilaterally nephrectomized rats, containing 4 times more angiotensinogen, yielded more angiotensin than the other plasmas. This was confirmed using the normal plasma diluted to $1 / 2$. When it was incubated with various concentrations of renin, formation of angiotensin was less than that from the plasma of original concentration, even before angiotensinogen had been consumed, i.e. at the lower renin concentrations. Consideration of the reninangiotensinogen reaction as a first order reaction also indicates that angiotensin formation varies with the concentration of angiotensinogen, regardless of whether the latter is in excess or not. From the equation of the reaction, ${ }^{13}$

$$
(A)=\left(A_{0}\right) e^{-K t}
$$

where $(A)$ is concentration of angiotensinogen, $\left(A_{0}\right)$ is that at $t=0$, and $K$ represents the reaction constant for a given amount of enzyme; it is obvious that if $\left(A_{0}\right)$ varies, (A) will vary regardless of the constancy of $\mathrm{Kt}$. The results of Hoobler et al. $^{3 \prime}$ are accordingly not valid.

\section{Discussion}

Under usual test circumstances, in which animals are anesthetized by pentobarbital or anesthetized and treated by pentolinium, cardiovascular reactivity to pressor agents is not modified by nephrectomy. However, there may be some change underlying in the cardiovascular system, since there is a lessened blood pressure fall to depressor agents and when all neural innervation to the cardiovascular system is excluded by pithing and by administration of autonomic blocking agents, there is a slight but definite augmentation in cardiovascular reactivity to pressor agents. In the preparation, destruction of the central nervous system by pithing, blockade of transmission at the ganglia by pentolinium, at the adrenergic nerve ending by bretylium, and at the cholinergic receptor site by atropine were achieved. The results accord, in a sense, with the view ${ }^{14)-19}$ ) that cardiovascular reactivity to pressor 
agents is augmented by bilateral nephrectomy.

The effect of depressor agents has been limited to a negative report ${ }^{201}$ on the response to $\mathrm{ACh}$ determined in rabbits. Our results suggest a diminished cardiovascular reactivity after nephrectomy. Increased sensitivity to pressor agents and decreased sensitivity to depressor agents observed after bilateral nephrectomy may indicate the possible participation of a specific renal function inhibiting blood pressure rise. Grollman ${ }^{21}$ has suggested that the kidney is responsible for maintenance of normal blood pressure, and proved that bilateral nephrectomy results in hypertension. Considering the specific renal function which inhibits pressor responses, Krieger and Hamilton ${ }^{22)}$ examined cardiovascular reactivity in the dog maintained by peritoneal lavage for up to 6 days after bilateral nephrectomy, and found an augmentation of the reactivity to pressor stimuli.

The claim of Hoobler et al. ${ }^{21},{ }^{3}$ that, if angiotensinogen is already in excess of the amount of renin injected into the normal animal, it is not likely that a further increase in its concentration in the bilaterally nephrectomized animal would lead to an increased production of angiotensin and, therefore, to a greater pressor response to renin is not supported by our studies nor is it theoretically probable. Bing's recent work ${ }^{23}$ ! has based on the same and incorrect claim mistakenly. Their finding that rats receiving $2 \mathrm{ml}$. of plasma from rats nephrectomized 24 hours before showed a greater response to hog renin than the rat infused with $2 \mathrm{ml}$. of normal rat plasma ${ }^{2}$ can be explained by an increase in angiotensinogen in the blood stream. The phenomenon has also been confirmed in rabbits and between rabbits and rats. ${ }^{24}$ Diminution of the increased sensitivity to renin in the nephrectomized rats after cross circulation with the normal ${ }^{25)}$ can also be explained by dilution of the increased angiotensinogen with the normal blood.

These experiments suggest that augmented response to renin after bilateral nephrectomy is mainly due to the increased angiotensinogen in the blood, and is partly due to the increased cardiovascular reactivity to angiotensin. The increase in angiotensinogen is a result presumably of the removal of a source of renin by nephrectomy.

\section{SUMMARY}

(1) Cardiovascular reactivity, 16 to 28 hours after bilateral nephrectomy was determined in rats by obtaining the dose-blood pressure-response curve in order to avoid the vertical bias.

(2) Bilateral nephrectomy did not increase cardiovascular reactivity to pressor agents (angiotensin and norepinephrine) in rats anesthetized by pen- 
tobarbital or anesthetized and treated by pentolinium, a ganglion blocking agent.

(3) In pithed animals without anesthesia nephrectomy augmented slightly but definitely the response to pressor agents.

(4) Further climination of the neural innervation in pithed animals by administration of bretylium, an adrenergic neurone blocking agent, atropine, and pentolinium, also resulted in an increased response to angiotensin after bilateral nephrectomy.

(5) But it is inadequate to explain the marked augmentation of response to renin after nephrectomy only by the slight increase in response to angiotensin. Response to renin was markedly augmented in pithed animals as well as under usual condition.

(6) Bilateral nephrectomy diminished cardiovascular reactivity to depressor agents (acetylcholine, adenosine triphosphate and histamine).

(7) Angiotensinogen in the plasma increased 4 times more after bilateral nephrectomy. This is the main cause of marked augmentation in response to renin.

(8) The claim that, if angiotensinogen is already in excess, a further increase in its concentration would not lead to an increased production of angiotensin was denied experimentally and theoretically.

\section{References}

1. Tigerstedt, R. and Bergman, P.G.: Skand. Arch. Physiol. 8: 223, 1898.

2. Blaquier, P., Hoobler, S.W., Schroeder, J., Gomez, A., and Kreulen, T.: Am. J. Physiol. 203 : 339, 1962.

3. Hoobler, S.W., Schroeder, J., Blaquier, P., and Demerjian, Y.: Can. Med. Assoc. J. 90 : $227,1964$.

4. Trendelenburg, U. : Pharmacol. Rev. 15:225, 1963.

5. Page, I.H. and Bumpus, F.M.: Physiol. Rev. 41 : 331, 1961.

6. Gross, F.: Arch. Exptl. Pathol. Pharmakol. 245 : 196, 1963.

7. Shibayama, F., Sokabe, H., and Sakai, F.: Arch. Intern. Pharmacodyn, 153: 334, 1965.

8. Sakuma, A.: Personal communication.

9. Kawabe, K. and Sokabe, H. : Jap. Heart J. 6: $51,1965$.

10. Muñoz, J.M., Braun-Menéndez, E., Fasciolo, J.C., and Leloir, F. : Am. J. Med. Sci. 200 : $608,1940$.

11. Collins, D.A. and Harakal, C.D.: Circulat. Res. 2 : 196, 1954.

12. Fasciolo, J.C.: Brit. J. Pharmacol. $21: 250,1963$.

13. Plentl, A.A. and Page, I.H.: J. Exp. Med. 78: 367, 1943.

14. Suganuma, Y.: Folia Pharmacol. Japon. $18: 159,1934$.

15. Page, I.H. and Helmer, O.M. : J. Exp. Med. 71 : 495, 1940.

16. Braun-Manéndez, E., Fasciolo, J.C., and Houssay, B.A.: Schwciz. Med. Wochschr. 71 : $280,1941$.

17. Houssay, B.A. and Dexter, L. : Ann. Int. Med. $17: 451,1942$.

18. McCubbin, J.W. and Page, I.H.: Circulat. Res. 2 : 35, 1954. 
19. Vanata, J.C., Danhof, I., and McMullen, M. : Am. J. Physiol. 185 : 167, 1956.

20. Billi, A. : Boll. Soc. Ital. Biol. Sper. 8 : 1760, 1933 (Abstr. in Chem. Abstr. 28 : 44841, 1934).

21. Grollman, A. : In: Hypertension, Humoral and Neurogenic Factors (edited by Wolstenholme, G.E.W. and Cameron, M.P.), Boston, Little, Brown : 122, 1954.

22. Krieger, E.M. and Hamilton, W.F. : Am. J. Physiol. 194 : 268, 1958.

23. Bing, J.: Acta Pathol. Microbiol. Scand. 60:312, 1964.

24. Takeda, T.: Personal communication.

25. Gross, F., Buschor, O., and Zcugin, H. : Am. J. Physiol. 202 : $1095,1962$. 